\title{
Principles of Collisional Activation in Analytical Mass Spectrometry
}

\author{
Scott A. McLuckey \\ Analytical Chemistry Division, Oak Ridge National Laboratory, Oak Ridge, Tennessee, USA
}

Collisional activation has played an essential role in the development of mass spectrometry/mass spectrometry (MS/MS). It was the first activation method to be employed and continues to be by far the most widely used. As instrumentation for MS /MS has evolved it has been found that collisional activation can be effected under a remarkably wide range of conditions for a wide range of ions. It is fair to conclude from the growth of MS MS over the past fifteen years that collisional activation has been spectacularly successful. However, it has limitations. As a community, we have learned much over the years regarding these limitations both from empirical and fundamental points of view. This overview provides background on the development of collisional activation and discusses the importance of the interaction potential and timing on mechanisms for energy transfer. Parts of the discussion is devoted to changing reference frames from the laboratory to the center of mass to simplify visualizing what is possible and what is probable in collisional activation. (J Am Soc Mass Spectrom 1992, 3, 599-614)

$\mathrm{T}$ This overview is based on a presentation made at the 1991 Sanibel meeting on ion activation and dissociation. The objective of that presentation was to provide a summary of the important mechanisms of collisional activation (CA) of polyatomic ions as it is currently effected. This overview shares that objective. The reader is also encouraged to refer to previous reviews [1-9] for more detailed coverage of various aspects of the topic. Because most interest in $\mathrm{CA}$ of polyatomic ions derives from its role in mass spectrometry/mass spectrometry (MS/MS), this discussion is given from an analytical perspective and is aimed at the practitioner of MS/MS. It should be explicitly recognized that the appearance of the MS/MS spectrum is only partly determined by the activation method and the dissociation behavior of the ion. Constraints imposed by the instrumentation (collection efficiencies, time frames, detector response, etc.) also play important roles. In practice, it is often difficult to either correct or fully account for instrumental effects when comparing MS/MS spectra obtained under different $C A$ conditions or from different instruments. Despite their importance, it is beyond the scope of this review to cover either unimolecular dissociation or instrumental discrimination effects. Nevertheless, if the objective of this review is met, the reader will appreciate both what is possible and what is probable in terms of $\mathrm{CA}$. This understanding facilitates the interpretation

Address reprint requests to Scott A. McLuckey, Analytical Chemistry Division, Oak Ridge National Laboratory, Oak Ridge, TN 37831-6365. of differences in MS/MS spectra acquired under different conditions or with different instruments.

The overview begins by defining the scope of the discussion and follows with a little history. An exhaustive review is not intended; the goal here is to give a feel for where we have been and how we have come to our current broad range of conditions that lead to CA. Then some analytical considerations are addressed in terms of activation method "figures of merit" to see how CA measures up. A minimal amount of the underlying physics is then introduced into the discussion. These sections will not be particularly useful for the devotee of collision theory, but they may be the most valuable part of the discussion for many readers. The objective here is to provide sufficient background to understand the language and issues of the CA literature. This background is then followed by a review of the major CA methods so far identified along with illustrative data drawn from the literature. The bulk of the discussion is devoted to single collision events. In practice, however, most MS/MS experiments are carried out under multiple collision conditions. This situation is therefore discussed, probably much too briefly, at the end of the overview, along with some musings on the prospects of $\mathrm{CA}$ in the challenges it faces for high mass ions.

\section{Scope}

Collisional activation is almost always mentioned along with collision-induced dissociation (CID) or the equiv- 
alent term, collisionally activated decomposition (CAD). In doing so, the assumption is usually made that CID proceeds in two steps, namely, collisional activation,

$$
Q+m_{p}^{+}+N \rightarrow m_{p}^{+*}+N^{\prime}
$$

where $Q$ is the change is kinetic energy of the system, $m_{f}^{+}$and $N$ are the precursor ion and target in their precollision states, and $m_{p}^{{ }^{*}}$ and $N^{*}$ are the collision par'ners in their postcollision states, followed some time later by unimolecular dissociation,

$$
m_{p}^{+^{*}} \rightarrow m_{a}^{+}+m_{b}
$$

where $m_{a}^{+}$and $m_{i b}$ are products of the unimolecular dissociation of $m_{p}^{+}$. CID need not proceed in two steps, however. A so-called stripping mechanism, whereby a part of the ion is tom away or knocked off in the course of the collision, might also prevail. In such a mechanism, collision and dissociation cannot be divorced so that CID would be more properly written as

$$
Q+m_{p}^{+}+N \rightarrow m_{a}^{+}+m_{b}+N^{\prime}
$$

The stripping mechanism is almost never invoked to explain CID spectra of polyatomic ions. Why not? For one reason, virtually all $\mathrm{CID}$ spectra are consistent with having involved unimolecular disseciation. For example, CID spectra of radical cations are, as a rule, qualitatively similar to the corresponding mass spectra from the neutral counterpart following electron ionization or photoionization, neither of which can involve an analogous stripping mechanism. Furthermore, it would be difficult to rationalize how dissociations involving complex rearrangements, which are often observed in CD spectra, could arise from a stripping mechanisn: In short, there is no clear evidence that CID effected under the conditions used in today's tandem mass spectrometers proceeds to any significant extent via a stripping mechanism. Virtually all of the CID data, however, are consistent with the two-step mechanism. For these reasons, the discussion is restricted here to the reaction in eq 1 , although the possibility for a stripping mechanism under some conditions is recognized.

In fact, a wide variety of reactions can occur under conditions often used to effect $C A$. Under certain conditions, they may compete with $\mathrm{CA}$, and the products of these reactions sometimes, though not always, appear in CID spectra. These include, for example, ion-niolecule reactions and charge-changing reactions. These reactions are not directly addressed here, although many of the generalizations presented for the reaction in eq 1 apply to any ion-target reaction.

\section{History of CA in Analytical Mass Spectrometry}

Collisional activation has a long history in mass spectrometry. In fact, in the earliest mass spectra Thomson observed signals that arose from CID [10], although Aston was the first to identify the process [11]. The evolution of $\mathrm{CA}$ as an ion structural tool, however, can be traced directly to work published in 1968 from the laboratories of McLafferty [12] and Jennings [13] (with due respect to a few prior CID studies (14-161). Much of the ensuing work was devoted to developing $\mathrm{CA}$ as a tool for distinguishing isomeric ion structures. McLafferty's group largely pioneered this application and performed many of the seminal studies of keV energy CA [17-19]. The work from the laboratory of Beynon and Cooks on various aspects of gaseous collisions was also highly influential [20-23]. Certainly the most significant factor in the growth of interest in CA came as MS/MS was recognized as a powerful tooi for mixture analysis. Cooks and co-workers were particularly prominent in the promotion of MS/MS in crude mixture analysis with the combination of chemical ionization and a BE geometry mass spectrometer [24, 25]. In the late 1970s, commercial instruments designed for MS/MS and CA became available.

Until the advent of the triple quadrupole instrument for analytical MS/MS, as described by Yost and Enke in the late 1970s [26, 27], all CA experiments were carried out on sector mass spectrometers. Virtually all work involved collision energies in the $\mathrm{keV}$ range, small atomic or diatomic targets such as helium, argon, or nitrogen, precursor ion masses less than $\mathbf{4 0 0}$ $u$, and target pressures sufficient to give one to ten collisions. The advent of the triple quadrupole mass spectrometer extended CA conditions into the laboratory collision energy range of 1 to $100 \mathrm{eV}$. The qualitative differences observed in CID spectra acquired with sector instruments and with triple quadrupole instruments generated interest in the underlying mechanisms of CA at low collision energies. Particularly noteworthy contributions to our understanding of $\mathrm{CA}$ at low collision energies were made by Douglas and Dawson $[6,28-30]$ in the early 1980 s.

A new wrinkle in CA conditions was added in 1982 when $\mathrm{CA}$ in an ion cyclotron resonance instrument was first executed by Cody and Freiser [31]. These experiments employed low collision energies, like the triple quadrupole instruments, but the path lengths available in the trapping instrument were orders of magnitude longer, as was the time between collisions. An even greater extension of CA conditions came with its application in the quadrupole ion trap operated with helium bath gas at 1 mtorr, first described by Louris et al. in 1987 [32]. The typical ion trap experiment employs collision energies less than $15 \mathrm{eV}$, but the number of ion-helium collisions can extend up to $10^{\text {t! }}$. The dynamics associated with each individual collision in the trapping instruments are not expected 
to differ from those associated with the collision in a beam experiment involving the same targets and collision energies. However, the significantly different technologies require rather different $C A$ conditions, which ultimately affect the CID spectra.

This capsule history makes no pretense at providing a balanced or comprehensive account of the development of CA in MS/MS. Rather, the purpose here is to point out the remarkable growth in the ways in which $\mathrm{CA}$ is effected. Life was fairly simple through most of the 1970 s when $\mathrm{keV}$ collisions ( $<10$ per ion) with inert gases and relatively small ions were the rule. Today, CA encompasses laboratory collision entrgies over the range of $<1 \mathrm{eV}$ to $10^{4} \mathrm{eV}\left(10^{5}-10^{6} \mathrm{eV}\right.$ can be accessed for highly charged ions in a sector instrument), collision number ranging from 1 to $10^{6}$, and both inert and reactive target gases. Collisional activation experiments are carried out today with sectors, hybrids (sector-quadrupole combinations), multiple quadrupoles, both magnetic and electrodynamic ion traps, and other mass analyzer combinations. Added to this diversity is the range in precursor ion masses (and charges) that can now be subjected to $C A$ thanks to the dramatic developments in ionization methods over thic pust decade. Little wonder that the role of CA in determining CID spectra is still not fully understood. There is so much to understand!

\section{Analytical Considerations}

Collisional activation is a means to an end. The end is the CID spectrum. Because most analytical applications of CA are focused on ion structure, it is of particular importance that the CID spectrum reflect the structure of the precursor ion. The CID spectrum for a given precursor ion is determined by a number of facto including the time frame of the experiment, instrumental discrimination effects, parent ion internal energy distribution after collision, and, in some cases, the partitioning of energ: within the ion. Some of these parameters are directly affected by the activation method. The analytical utility of any activation method can be assessed by considering its characteristics with respect to a set of figures of merit:

1. Details of the energy transfer distribution

2. Variability of the energy transfer distribution

3. Extent to which the reaction can be driven

4. Mechanism

The nature of the collisional energy transfer distribution, $P_{i}(\epsilon)$, plays a major role in the qualitative appearance of the CID spectrum. Under some CA conditions, for example, products from collisions requiring $Q$ values of $10 \mathrm{eV}$ can appear along with those involving $Q$ values of $<1 \mathrm{eV}$. Under other conditions, the width of $P_{c}(\epsilon)$ may be $<1 \mathrm{eV}$. To understand differences in CID spectra acquired under different conditions, it is therefore necessary to consider changes in $P_{i}(\epsilon)$ with collision conditions and possible instrumental effects. A number of studies have been performed to determine the details of $P_{c}(\epsilon)$ under Jifferent CA conditions [33-38]. For this discussion, it suffices to recognize that $P_{c}(\epsilon)$ is relatively broad at all but the lowest of collision energies and that it becomes broader as the collision energy increases. At the highest collision energies accessible in today's sector tandem mass spectrometers, the distribution is peaked at a most probable value of a few electron volts and shows a long high energy tail extending out to some tens of electron volts, at least for relatively small $m_{p}$.

The sensitivity of $P_{c}(\epsilon)$ to experimental conditions has important implications for the analyst. On the one hand, high sensitivity can make reproducibility problematic $[39,40]$. On the other hand, changes in CID spectra due to changes in $p_{c}(\varepsilon)$ can enhance the capability of $C A$ to distinguish ion structures [41]. On balance, it is highly desirable to be able to vary the eitergy transfer distribution (and preterably in a known fashion). Several approaches have been used in analytical applications of CA. A widely used approach is to operate under multiple collision conditions. This is a crude but very simple way to increase the total amount of energy deposited into the system. Experiments can be more readily interpreted and reproduced, however, when they are performed under conditions where only single collisions are likely [40].

$P_{c}(\epsilon)$ is most sensitive to changes in collision energy at low collision energies. Dissaciation of precursor ions as a function of internai energy can therefre be followed, at least qualitatively, by collecting CID spectra as a function of collision energy. This approach has been teferred to as energy resolved mass spuctrometry (ERMS) [41-44]. At high collision energies, large relative changes in collision energy become increasingly difficult to achieve, and, furthermore, $P_{\varepsilon}(\epsilon)$ tends to be very broad. Cooks and co-workers, however, have shown that portions of the $P_{i}(\epsilon)$ distribution at a fixed collision energy can be sampled by collecting products at selected scattering angles. This technique is referred to as angle resolved mass spectrometry (ARMS) [38, 41, 45-47]. Interpretation of ARMS data is complicated by the spread in product ion angular distribution due to energy released upon dissociation [38, 41, 48-51]. However, the physical phenorienon on which ARMS is based (see below) have been demonstrated to occur for polyatomic ions under the relevant $C A$ conditions [52-57], and the technique has been demonstrated to be useful in distinguishing isomeric ion structures, among other things [58-62].

A great strength of CA lies in the extent to which the reaction can be driven. This translates into analytically useful efficiencies. For beam-type MS/MS experiments, this point is illustrated by considering the Beer's 
law relationship

$$
I=I_{0} e^{-\sigma n l}
$$

where $I$ is the precursor ion signal after addition of the collision gas, $n$ is the target gas number density, and $l$ is the path length. (The product $n l$ is often referred to as "target thickness".) The cross section, $\sigma$, is for the sum of all loss processes for the precursor ion. This includes CID, scattering, and charge transfer to the target. The cross section for CID, $\sigma_{\mathrm{CID}}$, for any given ion depends on $P_{c}(\epsilon)$, the precursor ion internal energy content prior to collision, and the relevant internal energy dependent rate constants for dissociation. In practice, $\sigma_{\mathrm{CID}}$ ranges from 10 to $100 \AA^{2}$ for most ions, at least those of modest size. CA is therefore nonselective and is characterized by relatively large cross sections for a remarkably wide range of ions. It is also of practical importance that in beam-type instruments, target thickness is easily varied over many orders of magnitude by varying target gas pressure. In ion-trapping instruments, both target gas number density and path length can be varied to alter target thickness. For most other activation methods, either the cross section for activation is relatively small (e.g., photodissociation), or it is difficult to provide large number densities of activating agent (e.g., electron excitation), or both. Collisional activation has been a mainstay for analytical MS/MS largely because it is so easy to implement and because it is effective for a wide range of ions.

The mechanism by which energy is imparted into a precursor ion can also significantly affect the MS/MS spectrum. An important mechanistic question is, Can chemistry occur during the activation process? If the activation step takes place within a vibrational period, the ion cannot rearrange or otherwise react during the activation step. However, if activation proceeds in stepwise fashion, as in multiple collisions or multiphoton excitation [63], and the time between activation steps is long relative to vibration, reactions can occur during the activation process. Under single collision conditions, CA almost always occurs on the period of one vibration or less. Only at collision energies of a few electron volts or less and with targets of relatively high polarizability is the likelihood for the formation of a long-lived complex high. In such a case, chemistry can occur even under single collision conditions. Another mechanistic question is, In what form -electronic, vibrational, or rotational - is energy introduced into the precursor ion? If all ions behaved statistically-that is, if energy could transfer freely among all degrees of freedom prior to dissociation-this question would not be important. Many ions apparently do tend to behave statistically. In these cases, regardless of what form of energy is initially introduced into the ion, dissociation comes predominantly from excited vibrational states of the electronic ground state. However, there have been cases in which the form of excitation has a significant effect on the products that appear in the MS/MS spectrum [64], suggesting nonstatistical behavior. As discussed below, CA can deposit energy in all forms, depending on conditions.

From the generalizations made so far, $\mathrm{CA}$ as an activation method can be described as universal and easy to implement. A relatively broad distribution of energies is deposited into the ion upon collision, particularly at high collision energies. This distribution is sensitive to collision energy, particularly at low collision energies. Portions of the distribution can be sampled by collecting product ions at selected scattering angles. Collisional activation may involve rapid energy deposition under single collision conditions and at short interaction times, or it might be a relatively slow process, as in multiple collisions or collisions involving the formation of a collision complex. For any given ion-target collision, a variety of energy transfer mechanisms are possible. The likelihood for each depends on collision conditions and the nature of the collision partners. The remainder of this discussion is devoted to the mechanistic questions and relates the major mechanisms so far identified as being important in the CA of polyatomic ions.

\section{Frames of Reference}

To this point we have used the term laboratory collision energy in describing CA. We must now change our frame of reference to make the dynamics of $\mathrm{CA}$ much easier to visualize. For most MS/MS experiments, the target gas is assumed to be at rest, and the laboratory collision energy is simply taken as the kinetic energy of the ion. The precollision fast projectile-resting target situation is shown schematically in Figure 1a, which indicates the position ( $x$ marks the spot) and velocity of the center of mass (com) of the collision partners, $\mathbf{v}_{\text {com- }}$ This is how we see the collision, as we, like the
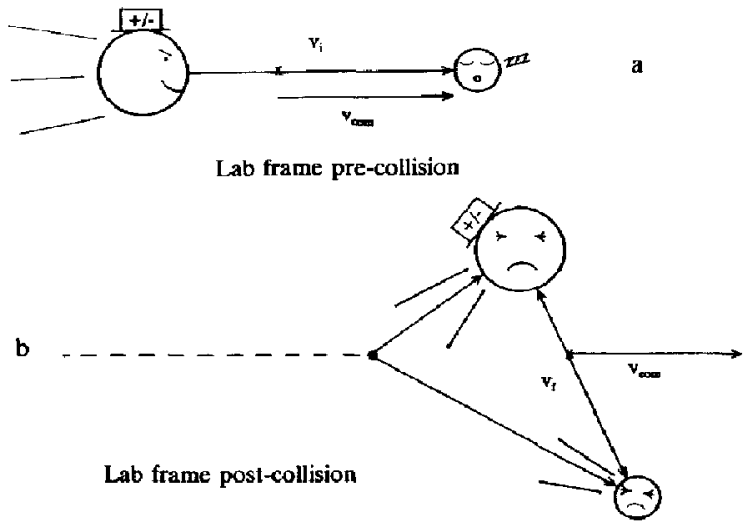

Figure 1. Cartoon depictions of the precollision (a) and postcollision (b) fast ion-resting target situation viewed in the laboratory frame of reference. 
target, are (approximately) at rest. The center of mass of the two collision partners is simply the point in space at which the mechanical moments of the two collision partners are equal and opposite:

$$
m_{p}\left(\mathbf{R}_{\mathrm{c}}-\mathbf{R}_{\mathbf{p}}\right)+N\left(\mathbf{R}_{\mathrm{c}}-\mathbf{R}_{\mathrm{N}}\right)=\mathbf{0}
$$

where $\mathbf{R}_{c}, \mathbf{R}_{\mathrm{p}}$, and $\mathbf{R}_{\mathbf{N}}$ are the instantaneous position vectors of the center of mass, of $m_{p}^{+}$, and of $N$, respectively, in the laboratory frame of reference. Solving for $\mathbf{R}_{\mathbf{c}}$ and differentiating with respect to time gives $\mathbf{v}_{\text {com }}$, the velocity vector for the center of mass:

$$
\mathbf{v}_{\mathrm{com}}=\mathbf{R}_{\mathrm{c}}^{\prime}=\frac{m_{p} \mathbf{R}_{\mathbf{p}}^{\prime}+N \mathbf{R}_{\mathbf{N}}^{\prime}}{m_{p}+N}
$$

The numerator is the total linear momentum, which is constant in the absence of an external field, and the denominator is the total mass, which is also conserved. Thus $\mathbf{v}_{\text {com }}$ is constant, both in magnitude $\mathbf{v}_{\text {com }}$ and in direction. The outcome of the collision is constrained by the conservation laws. For a binary collision in the absence of an external field, the collision partners are constrained to the plane defined by the initial velocity vectors, and the motion of the center of mass is conserved. In the laboratory frame of reference, the motions of the post collision products are superimposed on the motion of the center of mass of the collision pair. This is illustrated in Figure 1b, which shows one possible collision outcome. Note that the magnitude and direction of $\mathbf{v}_{\text {com }}$ are unchanged. Since this must be so, we can factor this motion out to give a clearer picture of the outcome of the collision. This is equivalent to "sitting" on the center of mass of the system and watching the collision partners approach each other from opposite directions prior to collision and recede from each other, again in opposite directions, after they have interacted. The pre- and postcollision cases depicted in Figure 1 are shown in Figure 2 in the frame of reference of the center of mass.

We know that the total energy of the system must be conserved and that the kinetic energy of the center of mass of the system is conserved. The difference, therefore, is the maximum energy that can be converted

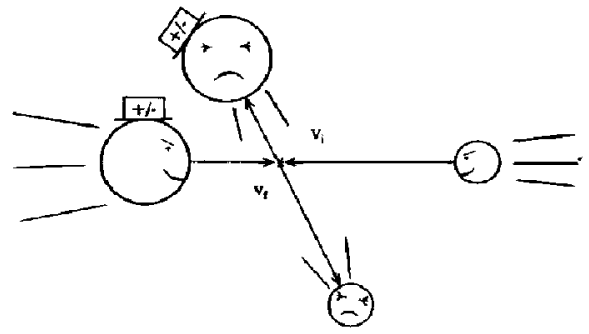

Figure 2. Cartoon depiction of the same collision shown in Figure 1 viewed in the center-of-mass frame of reference. between internal and translational modes of the collisional partners and is equal to the relative kinetic energy of the collision partners, $\mathrm{KE}_{\mathrm{rel}}$, given by

$$
\mathrm{KE}_{\mathrm{rel}}=\frac{1}{2} m_{p}\left(\mathbf{r}_{\mathbf{p}}^{\prime}\right)^{2}+\frac{1}{2} N\left(\mathbf{r}_{\mathrm{N}}^{\prime}\right)^{2}
$$

where $\mathbf{r}_{\mathbf{p}}^{\prime}$ and $\mathbf{r}_{\mathbf{N}}^{\prime}$ are the ion and target velocities in the center-of-mass reference frame. These velocities can be expressed as

$$
\begin{array}{r}
\mathbf{r}_{\mathrm{p}}^{\prime}=\mathbf{R}_{\mathrm{p}}^{\prime}-\mathbf{R}_{\mathrm{c}}^{\prime}=\mathbf{R}_{\mathrm{p}}^{\prime}-\mathbf{v}_{\mathrm{com}}=\frac{N \mathbf{v}_{\mathrm{rel}}}{m_{p}+N} \\
\mathbf{r}_{\mathbf{N}}^{\prime}=\mathbf{R}_{\mathbf{N}}^{\prime}-\mathbf{R}_{\mathrm{c}}^{\prime}=\mathbf{R}_{\mathbf{N}}^{\prime}-\mathbf{v}_{\mathrm{com}}=\frac{-m_{p} \mathbf{v}_{\mathrm{rel}}}{m_{p}+N}
\end{array}
$$

where $\mathbf{v}_{\text {rel }}=\left(\mathbf{R}_{\mathrm{p}}^{\prime}-\mathbf{R}_{\mathrm{N}}^{\prime}\right)$ and the expression obtained for $v_{\mathbf{c o m}}$ has been incorporated. Substituting for $r_{p}^{\prime}$ and $\mathbf{r}_{\mathbf{N}}^{\prime}, \mathrm{KE}_{\mathrm{rel}}$ can therefore be expressed as

$$
\mathrm{KE}_{\mathrm{rel}}=\frac{1}{2}\left(\frac{m_{p} N}{m_{p}+N}\right) \mathbf{v}_{\mathrm{rel}}^{2}
$$

The net exchange between internal energy and kinetic energy is reflected in $Q$, the change in the kinetic energy of the system. Equation 10 applies both before and after the collision, so that $Q$ is given by

$$
Q \equiv \Delta \mathrm{KE}_{\text {system }}=\frac{1}{2}\left(\frac{m_{p} N}{m_{p}+N}\right) v_{i}^{2}-\frac{1}{2}\left(\frac{m_{p} N}{m_{p}+N}\right) v_{f}^{2}
$$

where $v_{i}$ and $v_{f}$ are the pre- and postcollision relative velocities of the collision partners, respectively, and $m_{p} N /\left(m_{p}+N\right)$ is the reduced mass of the system. The maximum value of $Q$ is obtained when the second term of eq 11 is zero, that is, when the collision partners "stick together." $Q_{\max }$, therefore, is given by

$$
Q_{\max }=\left(\frac{N}{m_{p}+N}\right)\left(\frac{1}{2} m_{p} v_{i}^{2}\right)
$$

which is, of course, equal to $\mathrm{KE}_{\text {rel }}$. Note that in the fast ion-stationary target situation depicted in Figure 1a, $\frac{1}{2} m_{p} v_{i}^{2}$ is simply the laboratory collision energy, $\mathrm{KE}_{\mathrm{lab}}$ which leads to the commonly encountered expression

$$
\mathrm{KE}_{\mathrm{rel}}=Q_{\max }=\left(\frac{N}{m_{p}+N}\right) \mathrm{KE}_{\text {lab }}
$$

Figure 2 depicts a hypothetical center-of-mass reference frame precollision situation in which the ion approaches from the left and the target approaches from the right. What constraints are placed on the postcolli- 
sion velocities of the collision partners by the conservation laws? For an elastic collision $(Q=0)$, all of the possible postcollision velocities of the ion and the target can be visualized by rotating Figure 2 by $360^{\circ}$ about the center of mass. Circles are thereby inscribed for the possible postcollision velocities of the ion and target, respectively, as shown in Figure 3. These circles are referred to as the elastic circles for the ion and the target. Figure 3 is an example of what is referred to as a Newton velocity diagram $[2,65]$. For an inelastic collision $(Q<0)$, the postcollision velocity vectors of the ion and the target can fall anywhere inside the respective elastic circles provided they conserve momentum in doing so. That is, their momentum vectors must remain equal and opposite. Conversely, for a superelastic collision $(Q>0)$, the postcollision velocity vectors fall outside the respective elastic circles, again with momentum conservation. In all three cases, the angle between the incoming and outgoing velocity vectors is the so-called center-of-mass scattering angle, $\theta_{\text {com }}$. Both $\theta_{\text {com }}$ and the laboratory scattering angle, $\theta_{\text {lab }}$, are depicted in Figure 3 for an inelastic collision, that is, one in which the postcollision velocity vectors fall within the respective elastic circles, involving forward scattering.

The Newton velocity diagram for the ion serves as a very useful template for presenting ion scattering data. A complication to scattering diagrams associated with CID, however, stems from the fact that product ion velocity and angular distribution must be monitored rather than those of the precursor ion, and the product ion velocity distribution is affected by kinetic energy released in dissociation $[38,39,48-51]$. Kinetic energy

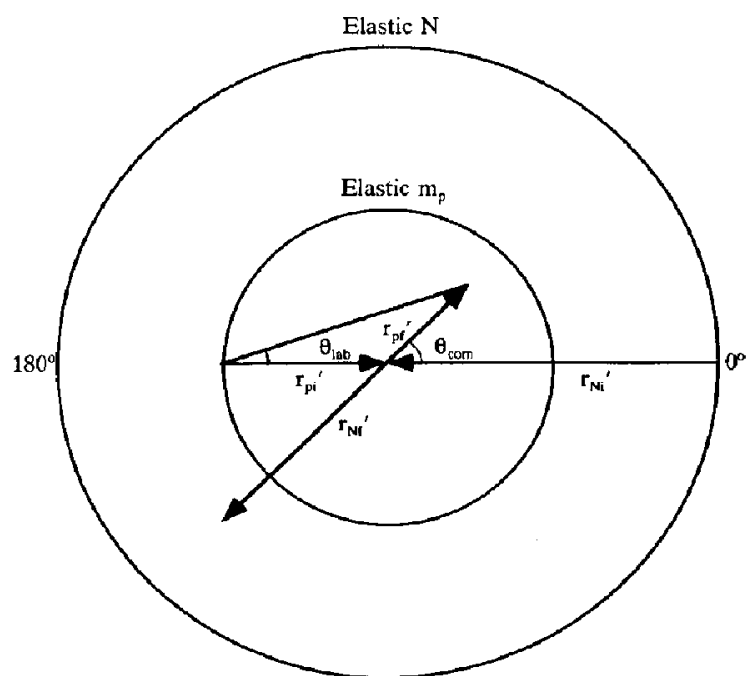

Figure 3. The Newton velocity diagram for the collision partners depicted in Figure 2. Pre- and postcollision velocity vectors for $m_{p}$ and $N\left(\mathbf{r}_{\mathbf{p i}}^{\prime}, \mathbf{r}_{\mathrm{pf}}^{\prime}, \mathbf{r}_{\mathrm{Ni}}^{\prime}\right.$, and $\mathbf{r}_{\mathrm{Ni}}^{\prime}$, respectively) are shown for an inelastic collision involving forward scattering at a nonzero scattering angle. release introduces a spread in velocities that may, in some cases, dominate the spread from precursor ion scattering. It is therefore easier to evaluate collision dynamics of CID for dissociations with relatively small releases of kinetic energy upon dissociation and, in particular, for dissociations in which the product ion is much more massive than the neutral product. This is simply due to the fact that a much smaller spread in velocities is introduced into the heavier fragment from kinetic energy release than into the lighter fragment.

The Newton velocity diagram illustrated in Figure 3 applies to what is sometimes called the "elastic limit" wherein the full mass of the precursor ion is involved in the collision. Some CA data have been interpreted as arising from a mechanism in which the target interacts with only a portion of the ion, the remainder of the ion playing the role of a spectator. This situation is referred to as the "binary limit." The elastic circle describing the binary limit can be determined from $m_{p}, N, v_{i}$, and the mass of the portion of the ion that undergoes collision $[28,49,66,67]$. The relative kinetic energy in the binary limit is given by

$$
\mathrm{KE}_{\text {rel }} \text { (binary) }=\frac{\mathrm{KE}_{\mathrm{lab}} N}{m_{p}+N\left(m_{a} / m_{p}\right)}
$$

where $m_{a}$ is the mass of the portion of the ion that undergoes elastic collision with the target. When the elastic circle that applies to a binary limit for any fraction of the precursor ion mass is plotted on the circle for the elastic limit, it always falls within the elastic limit and the "ion portion" target center-of-mass lies on the forward scattering side of the "full ion" target center of mass (i.e., the $0^{\circ}$ side).

Unfortunately, only a very few instruments have been built to allow the scattering diagram applicable to the CID of a polyatomic ion to be constructed [67-71]. Product ions must be collected as a function of mass, scattering angle, and kinetic energy from a well-defined scattering center and from a collision involving a well-defined $\mathrm{KE}_{\mathrm{rel}}$. Certainly no analytical MS/MS instruments can provide these capabilities. Why bother, then, with introducing the scattering diagram? Because some detailed chemical physics experiments have been performed involving polyatomic ion-target collisions that are highly revealing about the nature of the interactions, and they are most readily understood from the scattering diagrams. In particular, the recent work of Shukla and Futrell is designed to approach the problem of CA mechanism from the point of view of scattering dynamics [69, 71-74]. Their highly relevant findings are usually presented as ion intensity contours in scattering diagrams.

\section{Scattering and the Interaction Potential}

The elastic circle of the scattering diagram serves as the "canvas" to display the results of a collision exper- 
iment. Where the products actually fall on the diagram is determined (provided kinetic energy released in the dissociation is relatively small) by what happens at the scattering center, the center of mass of the system. In the frame of reference of the center of mass, the two collision partners approach from opposite directions, each with some kinetic energy. They recede from the center of mass after collision with a change in direction, if scattering occurs, and with changes in kinetic energy if the collision is either inelastic or superelastic. Where the products fall on the scattering diagram can provide important information about the nature of the ion-target collision. As indicated above, products that fall outside the elastic circle arise from a superelastic collision, and those that fall inside the elastic circle result from an inelastic collision. The angular distribution of the products can be used, in some cases, to correlate CID with the "closeness" of collision and in others to show that energy transfer proceeds through a long-lived collision complex. This section is intended to illustrate, in descriptive fashion only, how this information can be derived from the scattering diagram.

Of fundamental importance for any two-body collision is, of course, the potential energy as a function of the separation of the collision partners. A two-dimensional depiction of a generalized interaction potential is shown in Figure 4 for an atomic ion-atomic target system. This particular potential was plotted from an interaction potential of the form

$$
V(r)=A e^{-\Gamma r}-\frac{1}{2} \alpha e r^{-4}
$$

where $r$ represents the distance between $\boldsymbol{m}_{p}^{+}$and $N$. The first term in the expression represents the shortrange screened coulombic repulsion ( $A=7000 \mathrm{eV}$, $\Gamma=3.7 \AA^{-1}$ ), and the second term represents the long range ion-induced dipole attraction (polarizability, $\alpha$ $=6 \AA^{3}$ ). Other curves, which may apply for the ion and the target in different electronic states, are not shown in Figure 4. Furthermore, even for ground-state collision partners, the situation is much more complex for polyatomic ion-polyatomic neutral collisions. The

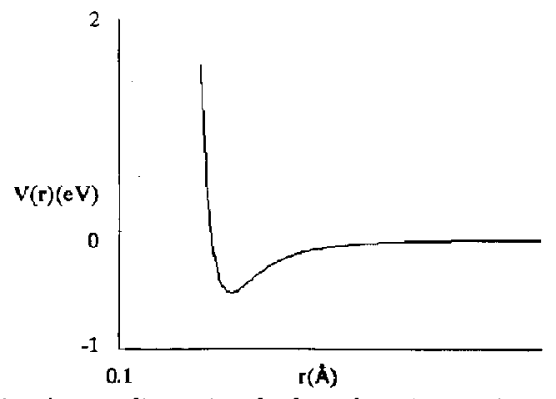

Figure 4. A two-dimensional plot of an interaction potential, $V(r)$, for an ion-target system as a function of ion-target separation, $r$. interaction potential, for example, can be dependent on the relative orientation of the collision partners. For our purposes it is sufficient to generalize that, at least qualitatively, the shape of the interaction potential for polyatomic ion-neutral collisions is similar to that for atomic collisions; that is, the collision partners repel at short distances and attract at long distances, the longterm attraction dominated by ion-dipole and ion-induced dipole terms.

We are accustomed to seeing interaction potentials plotted as in Figure 4, but it is perhaps easier to visualize scattering due to the interaction potential by viewing a three-dimensional representation of the interaction potential shown in Figure 5. (Note that the repulsive part of the potential is truncated at an arbitrary value.) Figure $5 b$, a tilted version of Figure $5 a$, shows precursor ion trajectories at three "impact parameters." The impact parameter $b$, an important variable in collision physics, is defined as the distance of closest approach of the two collision partners in the absence of an interaction potential. The significance of the impact parameter is that it can be used to calculate the classical trajectories of the ion and the target if $\boldsymbol{m}_{p}$ $N, V(r)$, and $\mathrm{KE}_{\text {rel }}$ are known. For our purposes, we simply note that small impact parameter collisions sample the repulsive part of the potential, whereas large impact parameter collisions tend to sample the long-range attractive part of the potential. It is also noteworthy that the impact parameter is proportional to the so-called reduced scattering angle [38, 49, 75,

a
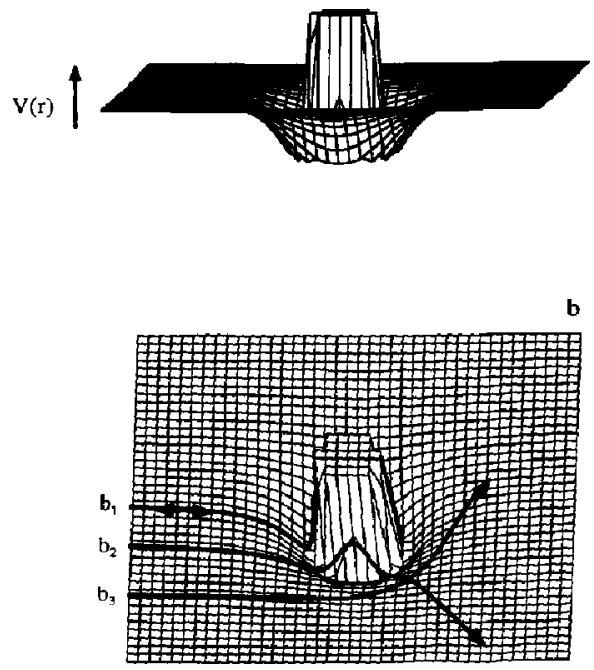

Figure 5. (a) A three-dimensional representation of an interaction potential. Note that $V(r)$ is truncated at an arbitrary value on the repulsive part of the surface. The potential energy of interaction is here assumed to be a function of only the intermolecular separation $r$ and is illustrated as a function of trajecto ries in the collision plane defined by the initial velocity vectors. (b) A tilted version of Figure 5a showing trajectories for three increasingly large impact parameters, $b_{1}-b_{3}$. 
76], that is,

$$
b \propto\left(\mathrm{KE}_{\mathrm{rel}} \theta_{\mathrm{com}}\right)^{-1}
$$

and that for small $Q$ and small $\theta_{\text {com, }}$

$$
\left(\mathrm{KE}_{\mathrm{rel}} \theta_{\mathrm{com}}\right)^{-1} \propto\left(\mathrm{KE}_{\mathrm{lab}} \theta_{\text {lab }}\right)^{-1}
$$

The ability to select some impact parameters from the entire range of impact parameters in acquiring MS/MS spectra is the basis for ARMS [38, 39, 45-47] in which certain values of $\theta_{\text {lab }}$ (typically $<2^{\circ}$ ) are selected at fixed $K E_{\text {lab. }}$.

The trajectories shown in Figure $5 b$ illustrate direct backscattering $\left(b_{1}\right)$, forward scattering due to a positive deflection from the repulsive part of the potential $\left(b_{2}\right)$, and forward scattering due to negative deflection from the attractive part of the potential $\left(b_{3}\right)$. Note that to conserve momentum, the trajectory of the target (not shown) in each case must be symmetrical with that of the ion with respect to $180^{\circ}$ rotation about the scattering center. It is also important to recognize that with a potential that is attractive at some values of $r$ and repulsive at others, deflection can occur from either part of the potential. By convention, scattering from the repulsive part of the potential is referred to as positive deflection and that from the attractive part is called negative deflection. Experimentally, we cannot distinguish between positive and negative deflection. However, the relative importance of positive versus negative deflection is highly dependent on conditions and $V(r)$. We can therefore anticipate the circumstances under which positive or negative deflection should dominate. As a rule, negative deflection increases with the absolute value of the "well depth," the minimum value of $V(r)$, and increases as $K E_{\text {rel }}$ decreases. Negative deflection is therefore maximized at low collision energies and with "sticky" (polarizable) targets. This is illustrated in the approximate expression for the classical rainbow angle, $\theta_{\text {Rcom }}$, the maximum negative deflection,

$$
\theta_{\mathrm{Rcom}} \approx \frac{c(\text { well depth })}{\mathrm{KE}_{\mathrm{rel}}}
$$

where the constant $c$ is on the order of 2 [77]. For example, consider two MS/MS experiments with a precursor of $m_{p}=200 \mathrm{u}$. If we use helium as the target, the well depth will be on the order of $0.1 \mathrm{eV}$. At a $\mathrm{KE}_{\text {lab }}$ of $5000 \mathrm{eV}, \theta_{\mathrm{Rcom}}$ would be roughly $0.1^{\circ}$, so that ions scattered beyond this value can be attributed to collisions involving interaction on the repulsive part of the potential. If we were to use ammonia as the target, the well depth would probably be on the order of $1 \mathrm{eV}$ [78]. At a $\mathrm{KE}_{\text {lab }}$ of $20 \mathrm{eV}, \theta_{\mathrm{Rcom}}$ would exceed $45^{\circ}$. It is clear, therefore, that at low collision energies, significant scattering from the attractive part of the potential can occur with sticky targets. Indeed, as we shall see, the ion and the target may stick together long enough to give a full rotation or more (scattering angles greater than $360^{\circ}$ ). This is simply the orbiting collision situation encountered with thermal energy and "near thermal" energy ion-molecule reactions.

\section{Timing}

To this point we have indicated how the conservation laws place certain limits on the outcome of a binary collision, and we have changed our frame of reference to that of the center of mass of the system to clearly see what is possible. We have also discussed the interaction potential and its effect on where products are likely to fall at different impact parameters and at different collision energies, that is, to enable discussion of what is probable. We now introduce the importance of timing in collisional energy transfer. The time over which the ion and the target interact, the so-called interaction time, plays a major role in determining the nature (vibrational versus electronic) and mechanism of excitation. The role of timing is ordinarily presented within the context of "Massey's adiabatic criterion" [79] as discussed below.

In qualitative terms, Massey's adiabatic criterion states that the probability for energy transfer into a particular mode is maximized when the interaction time is roughly equal to the mode's effective period of motion. This is often couched in terms of the Massey parameter, $t_{c} / \tau$, where $t_{c}$ is the interaction time and $\tau$ is the effective period of motion. The interaction time is normally determined as an interaction distance (usually taken as $4-10 \AA$ [80]) divided by the relative velocity of the collision partners. At Massey parameters much greater than 1, the collision is very slow relative to the effective period of motion so that the ion-target system can adjust adiabatically, that is, without a change in state, to the perturbation. In this case, a transition is unlikely. At Massey parameters much less than 1, the interaction is too short for the internal motion, again resulting in little likelihood for excitation. The coupling of translation with an internal motion is therefore maximized near "resonance," that is, when the forces induced by the collision are changing on the same time frame as that of the internal motion.

Within the context of the Massey adiabatic criterion, the relative velocity at which the probability for an inelastic transition is maximized is approximated by

$$
v_{\max } \approx \frac{a(\Delta E)}{h}
$$

where $a$ is the effective distance of interaction, $h$ is Planck's constant, and $\Delta E$ is the energy difference between states [80]. (It is important to recognize that the values of $a$ and $\Delta E$ for a transition between two 
electronic states may differ when the transition takes place at long separations, wherein the state levels are little perturbed by the collision, versus when the transition takes place at an avoided crossing between the states brought close together in the collision complex [1].) In instances in which radiationless transitions between electronic states is rapid $\left(\tau \approx 10^{-15} \mathrm{~s}\right), v_{\max }$ falls in the $10^{4} \mathrm{eV}$ collision energy range for relatively small $m_{p}$ and in the hundreds of thousands to low millions of electron volts for the more massive ions $\left(m_{p}>1000\right.$ u) commonly encountered today. This is illustrated in Figure $6 \mathrm{a}$, which shows a plot of the $\mathrm{KE}_{\text {lab }}$ corresponding to $v_{\max }$ as a function of $m_{p}$ for an excitation $(\Delta E)$ of $2 \mathrm{eV}$ and an effective distance of interaction of $10 \AA$. It is clearly apparent that in the fast ion-stationary target situation relevant to most MS/MS instruments, laboratory kinetic energies fall well short of those that maximize the probability for electronic excitation of this sort. There is, however, an exponential drop-off of cross section as the ion velocity falls below $v_{\max }$ so that, at least for smaller precursor ions, fast electronic transitions can occur with low but nonzero probability in the kiloelectron-volt collision energy range. For direct excitation of vibrational modes $\left(\tau=10^{-14}-10^{-13}\right.$ s), the laboratory collision energy at the $v_{\max }$ for $\Delta E=$ $0.1 \mathrm{eV}$ with $a=10 \AA$ as a function of $m_{p}$ is shown in Figure $6 \mathrm{~b}$. The range of laboratory collision energies in this case falls within the range accessible to many analytical tandem mass spectrometers. However, en-
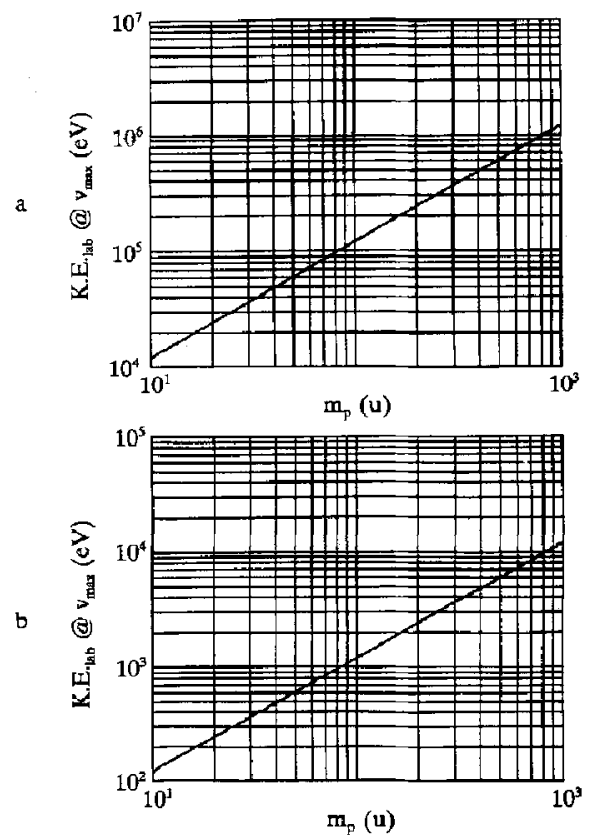

Figure 6. Log-log plots of $\mathrm{KE}_{\mathrm{lab}}$ at $v_{\max }$, as determined from eq 13 for $a=10 \AA$, as a function of $m_{p}$. (a) $\Delta E=2 \mathrm{eV}$; (b) $\Delta E=0.1$ eV. ergy transfers of a few tenths of an electron volt cannot account for most CID (see below).

\section{Mechanisms of Collisional Activation}

A variety of mechanisms are possible for the transfer of energy from translation ( $\mathrm{T}$ ) to internal modes in polyatomic ion-neutral target collisions under the range of conditions used in today's tandem mass spectrometers. Most of these mechanisms were described in the seminal paper by Durup [1], in which the mechanisms of CID of diatomic ions received heavy emphasis. In this section we classify CA mechanisms based on the nature of excitation-vibrational (V), electronic (E), and combined vibrational-electronic. (Rotation is grouped with vibration.) Each mechanism is discussed and, in some cases, illustrated with data taken from the literature presented in the form of the Newton velocity diagram described earlier.

$T \rightarrow V$

Complex formation. The most efficient form of translational energy-to-internal energy conversion is via the formation of a long-lived complex between the ion and the target (Durup's "process 3"). Orlando et al. have exploited this characteristic of complex formation in their use of endothermic ion-molecule reactions to effect CID of peptides [81]. Once the ion and the target undergo a capture collision (i.e., they "stick" together), all of the $\mathrm{KE}_{\mathrm{rel}}$ is present in the complex. To fit our working picture of $\mathrm{CID}$ as a two-step process, the complex must break up into the ion and the target once again before the ion dissociates. (However, the complex may dissociate into other primary products. In this case, we might call the process an ion-molecule reaction to differentiate it from a reaction that simply adds energy into the precursor. In the former case, dissociation reflects precursor ion structure, whereas in the latter case, dissociation reflects the structure of the ion-target complex.) The fraction of $\mathrm{KE}_{\text {rel }}$ that eventually finds itself in the precursor ion tends to increase with the lifetime of the ion-target complex, with the total number of degrees of freedom of the complex, and with the fraction of the total number of degrees of freedom of the complex present in the ion. The lifetime of the complex increases with the ion-target well depth and the number of degrees of freedom in the complex and decreases rapidly with $\mathrm{KE}_{\mathrm{rel}}$ [82]. Experimentally, therefore, complex formation is maximized by use of a polarizable (sticky) polyatomic target and very low $\mathrm{KE}_{\mathrm{rel}}$. The latter condition constitutes the major drawback to the use of complex formation for CA under single-collision conditions in that, although the efficiency $\left(Q / \mathrm{KE}_{\mathrm{rel}}\right)$ is high, $\mathrm{KE}_{\mathrm{rel}}$ is ordinarily very low.

Under the conditions necessary for complex formation, the classical rainbow angle can be very large 
$\left(>360^{\circ}\right)$, and because the target interacts with the entire ion, the elastic limit applies to the product ions. No experimental Newton diagrams have yet been reported for CA via complex formation, but one for an ion-molecule reaction can serve to illustrate some of the points just mentioned. Figure 7 shows a Newton velocity diagram for the product ion $\mathrm{O}_{2} \mathrm{D}^{+}$from the reaction of $\mathrm{O}_{2}^{+.}+\mathrm{D}_{2}$ at $\mathrm{KE}_{\mathrm{rel}}=2.76 \mathrm{eV}$. Note that the product ion distribution is symmetric with respect to reflection about the line passing through the center of mass and $90^{\circ}$ and $-90^{\circ}$ deflection [84]. The high efficiency and the symmetric distribution are both consistent with the formation of a long-lived complex, one that could rotate at least $360^{\circ}$ prior to breakup. In terms of the interaction potential of Figure 5 , we can envision this situation as a large impact parameter trajectory showing negative deflection to the point that the arrow bends completely around the center of mass.

The high efficiency associated with complex formation is hardly surprising when viewed as a unimolecular energy redistribution situation. Energy deposited into a bond is known to significantly dissipate throughout a molecule within tens of vibrations [85]. How can we reconcile the Massey criterion with the increase in efficiency with lifetime? Highest efficiencies are observed when the ion and target are in intimate contact for rotational periods yet vibrational energy transfer is efficient. There is really no discrepancy here when it is recognized that the ion and target "collide" many times at vibrational frequencies during the life of the orbiting complex. The Massey parameter for each "collision" is quite favorable $\left(t_{c} / \tau=1\right)$. Complex formation can therefore be regarded as the precursor ion undergoing multiple collisions with the same target.

Impulsive collision. A particularly important mechanism for CA, perhaps the most important over a wide

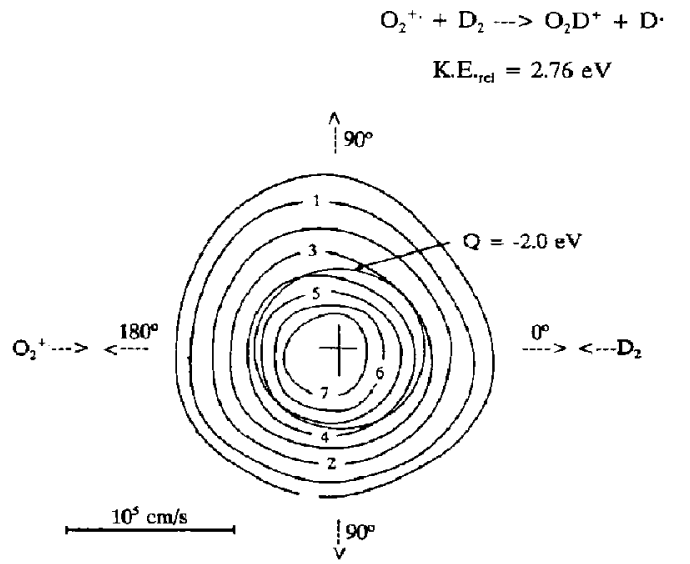

Figure 7. Newton diagram for the $\mathrm{O}_{2} \mathrm{D}^{+}$product ion from the reaction $\mathrm{O}_{2}^{+}+\mathrm{D}_{2} \rightarrow \mathrm{O}_{2} \mathrm{D}^{+}+\mathrm{D}$ at $\mathrm{KE}_{\text {rei }}=2.76 \mathrm{eV}$. Product ion velacity contours are shown plotted on an inelastic circle of $Q=-2.0 \mathrm{eV}$ for reference. (Adapted from ref 83 .) range of commonly encountered MS /MS conditions, is the so-called impulsive or binary collision mechanism $[28,49,66,67,83]$ referred to as "process 2 " in Durup's paper [1]. This mechanism involves an elastic collision between the target (or part of the target) and part of the ion with some of the recoil energy distributed into vibration. The repulsive part of the potential is sampled, resulting in momentum transfer (scattering). Product ions are therefore expected to show a significant off-axis component, which, at constant impact parameter, decreases as $\mathrm{KE}_{\text {rel }}$ increases (see Equation 16). Product ion velocities in the purely impulsive merhanism are bounded by the binary limit (see above). Shukla, Futrell, and co-workers have shown several Newton velocity diagrams of polyatomic ion CID that are consistent with the impulsive mechanism (involving positive deflection from the repulsive part of the interaction potential, Figure 5). Figure 8 shows, for example, a portion of the Newton diagram for $\mathrm{CH}_{3} \mathrm{CO}^{+}$from ionized acetone colliding with argon at $\mathrm{KE}_{\mathrm{rel}}=123 \mathrm{eV}$ [74]. The product ions show predominantly forward scattering with a cone of scattered intensity centered between $\theta_{\mathrm{com}}=1-4^{\circ}$. This behavior - nonzero angle forward scattering - has proved to be the rule in most of the studies reported to date by Futrell and associates at $\mathrm{KE}_{\text {rel }}$ in excess of several tens of volts up to hundreds of volts. These observations, and many others $\left[1,28,38,49,52,69,86^{-91}\right]$, indicate that impulsive collisions play an important role in CID over a large range of conditions. It is worth noting that the stripping mechanism mentioned earlier is also bounded by the binary limit. Product ions from a stripping mechanism would therefore be expected to fall within the same region of the Newton diagram as those from the impulsive collision. Their distinction may seem subtle-fragmentation during the course of collision (stripping) versus delayed unimolecular fragmentation (impulsive mechanism for $\mathrm{CA}$ ) - but the effect on the CID spectrum is important. As indicated above, although the stripping mechanism cannot be ruled out under all conditions, the preponderance of CID data suggests that fragmentation occurs significantly after collision.

$$
\begin{aligned}
& \mathrm{CH}_{3} \mathrm{COCH}_{3}^{++}+\mathrm{Ar}-->\mathrm{CH}_{3} \mathrm{CO}^{+}+\mathrm{CH}_{3}+\mathrm{Ar} \\
& \text { K.E. } \mathrm{rcl}_{\mathrm{rcl}}=123 \mathrm{eV}
\end{aligned}
$$

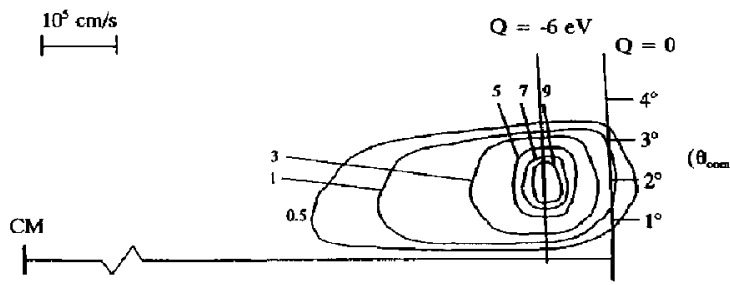

Figure 8. A portion of the Newton velocity diagram for the $\mathrm{CH}_{3} \mathrm{CO}^{+}$product ion from tonized acetone colliding with argon at $\mathrm{KE}_{\mathrm{rel}}=123 \mathrm{eV}$. (Adapted from ref 72.) 
The efficiency, $Q / \mathrm{KE}_{\text {rel, }}$ of the impulsive mechanism is typically much lower than that of complex formation. For one reason, the energy available for conversion into internal energy is smaller in the binary limit than in the elastic limit. Furthermore, only a single collision occurs in the impulsive mechanism, in contrast with the multiple collisions possible within a single complex formation event. However, $\mathrm{KE}_{\mathrm{rel}}$ can be orders of magnitude higher. In fact, the impulsive collision mechanism is the only mechanism involving vibrational excitation of the electronic ground state that can account for energy transfers in excess of 4 or 5 $\mathrm{eV}$ in a single collision, at least for $m_{p}<200 \mathrm{u}$. It is also noteworthy that the impulsive mechanism is relatively insensitive to timing at collision times on the order of a vibrational period and shorter. Since energy transfer does not take place by the direct excitation of a periodic motion but rather takes place by indirect excitation via an elastic collision, the impulsive mechanism becomes probable once the collision time is on the order of, and shorter than, the period of vibration. The impulsive mechanism is therefore likely over a wide range of MS/MS conditions and for a wide range of $m_{p}$. Derrick and co-workers, for example, have interpreted the $\mathrm{CA}$ data acquired for kiloelectron-volt $\mathrm{KE}_{\mathrm{lab}}$ precursor ions of $m_{p} \approx 10^{3} \mathrm{u}$ in collisions with helium as arising from impulsive collisions [87-89].

The impulsive mechanism may account, at least in part, for much of the ARMS data mentioned above. The trend in increasing energy deposition into the precursor ion when product ions are selected at increasing laboratory scattering angles is consistent with sampling smaller impact parameter collisions. Although the impulsive mechanism requires a small $b$ collision with part of the ion, the phenomenon of scattering does not necessarily imply that the impulsive mechanism is responsible. If the ion and the target behave as rigid structures, the collision would be elastic, at least vibrationally. Energy transfer could occur electronically, however, via curve crossing (see below). The postcollision precursor ion distributions for these two possibilities are bounded by the binary and elastic limits, respectively. Both mechanisms are expected to be important under some conditions. The relative importance of these mechanisms for collisional scattering over the range of CA conditions used in today's tandem mass spectrometers, however, is still poorly characterized.

Direct induction of vibration. Another classification of vibrational excitation is that induced by rapidly changing polarization forces during the collision, resulting in the excitation of one or more of the precursor ion vibrational modes. Such a mechanism is expected to be sensitive to timing because it involves direct excitation and is bounded by the elastic limit because it involves interaction of the entire ion with the target. It is also expected to be a large impact parameter mechanism. Russek described such a mechanism [92] to explain, in part, the CID behavior of $\mathrm{HeH}^{+}$. Because it is a longrange interaction, very little momentum transfer is expected. Energy transfers, however, are limited to a few vibrational quanta. The contribution of such a mechanism to CID of polyatomic ions is therefore probably limited to precursor ions already close to the dissociation limit. As indicated at the end of the discussion about timing, this type of mechanism cannot account for most CID observed in MS/MS.

\section{$T \rightarrow E$}

Vertical excitation. Durup referred to this mechanism as "process 1 " in which a vertical electronic transition occurs from a large impact parameter collision. Negligible scatter is expected, and the difference in electronic energy levels changes insignificantly during the collision. The $\Delta E$ term in eq 19 is therefore the spacing of the relevant electronic levels for a vertical FranckCondon transition at infinite ion-target separation. We would expect products from this mechanism to be greatly forward scattered and bounded by the elastic limit. This process is expected to be important only at short interaction times $\left(10^{-14} \mathrm{~s}\right.$ or less). In most MS $/ \mathrm{MS}$ experiments with present-day instrumentation, interaction times tend to be longer than $10^{-14} \mathrm{~s}$, particularly for $m_{p}$ values greater than a few hundred mass units. As illustrated in the discussion about timing, the relative velocity at which the cross section for a $2 \mathrm{eV}$ transition is maximized at precursor ion kinetic energies is significantly higher than most tandem mass spectrometers can provide. There is essentially no experimental evidence to indicate how important this mechanism is for the CID of large polyatomic ions in today's tandem mass spectrometers. Based on the Massey criterion, it can be ruled out over a wide range of conditions. However, it may be important for very light precursor ions ( $m_{p}<50 \mathrm{u}$ ) in the kiloelectron-volt collision energy range and is known to be so in the case of some diatomic ions [2].

Curve crossing. The mechanism most frequently credited for electronic transitions in the $\mathrm{CA}$ of polyatomics is the curve-crossing mechanism, wherein a net transition occurs at an avoided crossing along the ion-target coordinate. Figure 9 illustrates this situation. A reaction coordinate for the precursor ion is shown in one dimension, and the ion-target coordinate is shown orthogonal to it. Two electronic states of the precursor ion are shown which "cross" at some point along the ion-target coordinate. Timing is particularly important in this mechanism in that the collision must be slow enough to allow a transition to the upper state but fast enough to avoid crossing back down to the lower state as the ion and target recede from each other. The rate of change of the ion-target distance at the crossing point is therefore key. The Massey criterion applies here, the $\Delta E$ term being the difference in energies of the two states at the avoided crossing. 


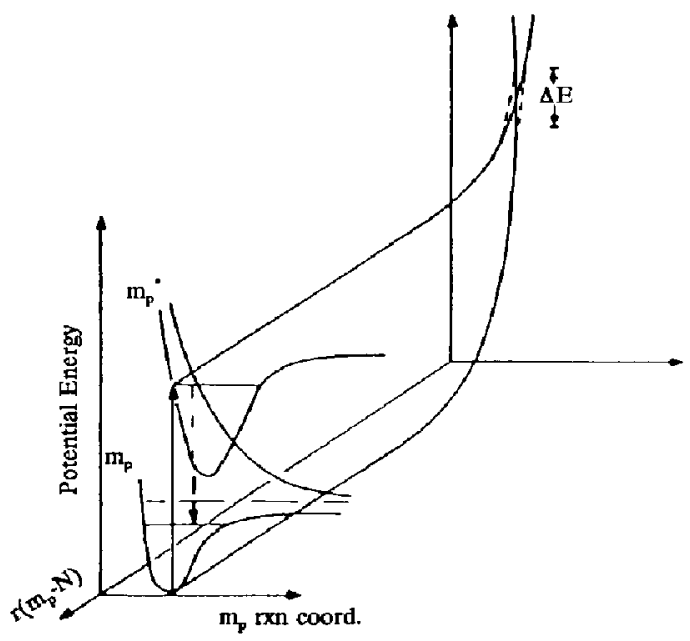

Figure 9. Schematic interaction potentials illustrating the curve-crossing mechanism for collisional activation. (Adapted from ref 4 .)

The curve-crossing mechanism is expected to be more probable at lower relative velocities than the vertical excitation mechanism due to the smaller value of $\Delta E$. It is also a smaller impact parameter process. Although both are bounded by the elastic limit, it is generally not possible to predict to what extent scattering will accompany curve crossing (whereas negligible scattering occurs for vertical excitation). Scattering associated with a given crossing will be determined by its location on the potential surface and the collision energy. Reid has recently described several studies involving "scatter profiling," a technique that allows the collisional scattering distribution to be extracted from the experimentally observed product ion angular distribution, for several small cations at kiloelectronvolt $\mathrm{KE}_{\mathrm{lab}}[53-57]$. The data presented so far appear to be consistent with scattering arising from curve crossing; that is, the product ion angular distributions appear to be consistent with scattering involving the entire precursor ion [57].

The curve-crossing mechanism is normally associated with fast interactions (high collision energies). A particularly interesting example of curve crossing on the repulsive part of the potential at low collision energies, however, comes from the elegant work of Futrell and co-workers on the CID of ionized acetone $[72,73]$. At a $\mathrm{KE}_{\mathrm{rel}}$ of $1.58 \mathrm{eV}$ with helium as the target, the Newton diagram of Figure 10 was acquired for the $\mathrm{CH}_{3} \mathrm{CO}^{+}$product ion from ionized acetone. Two maxima were observed on the backscattered side of the diagram at $Q$ values of $+2.2 \mathrm{eV}$ and $-1.3 \mathrm{eV}$, respectively. The signal observed at the $Q$ value of $2.2 \mathrm{eV}$ arises from a superelastic collision, a rather unusual source of CID. These workers have made a convincing case that this signal arises from the depopulation upon collision of a long-lived excited electronic state of ionized acetone present in the precursor ion beam. The

$$
\begin{gathered}
\mathrm{CH}_{3} \mathrm{COCH}_{3}^{+}+\mathrm{He}-->\mathrm{CH}_{3} \mathrm{CO}^{+}+\mathrm{CH}_{3}+\mathrm{He} \\
\text { K. } \mathrm{E}_{\text {rel }}=1.58 \mathrm{eV}
\end{gathered}
$$
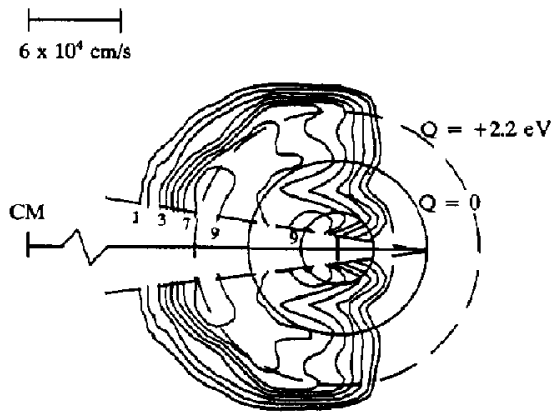

Figure 10. The Newton velocity diagram for the $\mathrm{CH}_{3} \mathrm{CO}^{+}$ product ion resulting from collisions of ionized acetone with helium at $\mathrm{KE}_{\mathrm{rel}}=1.58 \mathrm{eV}$. (Adapted from ref 74 .)

signal at $Q=-1.3 \mathrm{eV}$ represents the promotion of acetone ions in the ground electronic state (but with about $1 \mathrm{eV}$ of vibrational energy) into the excited state with subsequent dissociation. As required by microscopic reversibility, both processes occur over the same surface, which, in this case, involves a direct head-on (very small impact parameter) collision. Timing is very important in these reactions as they are no longer observed to be major reaction channels as the collision energy is raised further. The dynamics shift over to the mechanism (probably impulsive) responsible for the scattering diagram of Figure 8 .

The acetone results, and those recently obtained for ionized nitromethane [93] and benzene [94], are interesting in that $\mathrm{T} \rightarrow \mathrm{E}$ and $\mathrm{E} \rightarrow \mathrm{T}$ conversions due to curve crossing are observed at low collision energies (long $t_{c}$ ). Historically, the curve-crossing mechanism was believed to dominate at high collision energies (short $t_{c}$ ) due to Massey parameter considerations. These results indicate, however, that at least when "isolated" electronic states are involved, electronic transitions can occur at longer interaction times. The extent to which similar behavior might be observed for other types of ions of analytical interest, such as protonated species and negative ions, is currently unknown and constitutes an interesting line of investigation.

\section{Combined $T \rightarrow E, V$}

Curoe crossing + impulsive collision. Of course, vibrational excitation can accompany direct electronic excitation when an excited electronic state is populated in excited vibrational states due to the vertical nature of the transition. Furthermore, if radiationless transitions are fast, energy initially present in electron motion can find its way into vibration of the electronic ground state. These are regarded here as indirect $\mathrm{T} \rightarrow \mathrm{V}$ mechanisms. This category is concerned with direct vibrational excitation proceeding in concert with an elec- 
tronic transition. Such a combined mechanism results in what Durup referred to as an "oblique" or nonvertical transition. Such a transition can result from curve crossing to an excited electronic state in concert with an impulsive collision. This situation was alluded to briefly earlier and would be expected from curve crossing on the repulsive part of the potential for an ion that does not behave like a rigid structure. Various combinations of energy partitioning among the collision partners can occur. The ion may undergo both vibrational excitation and electronic excitation, the ion may be vibrationally excited while the target is promoted to an excited electronic state, and so on.

An oblique transition is possible over the range of conditions that both impulsive and curve-crossing mechanisms are likely. Recent ARMS data, for example, give evidence for both electronic and direct vibrational excitation in collisions resulting in nonzero scattering angles at kiloelectron-volt collision energies [38]. The contribution from electronic excitation appeared to decrease as the collision energy decreased. In light of the recent results for the "nonstatistical" ions obtained by Futrell and colleagues, however, oblique transitions may occur both at long and at short interaction times, depending on timing requirements for the electronic transitions.

Summary of mechanisms. In pulling together the discussion about mechanisms we must first consider the issue of how to characterize collision conditions. Frequently we speak in terms of $\mathrm{KE}_{\text {lab }}$ for convenience. Sometimes we speak (more correctly) in terms of $\mathrm{KE}_{\text {rel }}$ because we recognize the constraints imposed by the conservation laws. $\mathrm{KE}_{\text {rel }}$, however, indicates only what is possible, in terms of energy transfer; it says nothing about what mechanism or mechanisms and what efficiencies are likely. It would be more informative, therefore, to relate both $\mathrm{KE}_{\text {rel }}$ and $t_{c}$ [95]. We might also need to know, however, the number of degrees of freedom of the ion-target pair, the target polarizability, whether or not the ion behaves statistically, and so on. These characteristics are specific to the ion-target pair. We must therefore recognize that we are forced to paint with a very broad brush when we characterize single collision CA with $\mathrm{KE}_{\text {rel }}$ and $t_{c}$.

Collisional activation can proceed with sticky targets at low $\mathrm{KE}_{\mathrm{rel}}$ and long $t_{\mathrm{c}}$ via complex formation. At $t_{c}$ values on the order of a vibrational period and shorter, the impulsive mechanism appears to be important over the entire range of $\mathrm{KE}_{\mathrm{rel}}$ accessible in today's tandem mass spectrometers. Curve crossing associated with low impact parameter collisions can also occur for ions with isolated electronic states at $t_{c}$ on the order of a vibration. For fast electronic transitions, curve crossing is expected to be most important at $t_{c}$ less than vibrational periods, which normally implies relatively large $\mathrm{KE}_{\text {rel }}$. Vertical electronic excitation is also possible at short $t_{c}$, but relative velocities necessary to maximize the probability for this process are not accessible with present-day MS/MS instruments. The combined curve-crossing-impulsive collision mechanism resulting in "oblique" transitions can occur over the range of conditions that the individual mechanisms overlap.

\section{Target Effects}

Other than mentioning the use of "sticky" targets for complex formation and the role of target mass in determining $\mathrm{KE}_{\mathrm{rel}}$, we have said very little about the nature of the target on the dynamics of CID of polyatomic ions. This is due, in part, to the fact that different target effects may apply to each mechanism. The topic would constitute a rather lengthy story in itself. However, the main reason is that very little is known about the detailed role of the target on the dynamics of CID, at least not enough to be able to draw a reasonably complete picture. That is not to say that target effects in MS/MS have not been the focus of study. Indeed, a number of studies have been reported on the role of the target in energy deposition, scattering, charge exchange, and the like $[28,33,38,86$, 96-104] over a wide range of collision conditions. They have been very useful in establishing which targets to use under various CA conditions from the analytical standpoint. For example, light targets with relatively high ionization potentials, usually helium, are used in kiloelectron-volt $\mathrm{KE}_{\text {lab }} \mathrm{CA}$ of small to moderately sized precursor ions to minimize charge exchange and scattering. Relatively heavy, high ionization potential, atomic targets (e.g., Ar, $\mathrm{Xe}$ ) are used in electron-volt $\mathrm{KE}_{\text {lab }}$ collisions to minimize charge transfer and ionmolecule reactions and to maximize $\mathrm{KE}_{\mathrm{rel}}$. However, target effects have typically been studied by acquiring CID spectra, which are usually not sensitive to CA mechanism, particularly if the ion behaves statistically. CID spectra have been useful in cases where $P_{c}(\epsilon)$ reflects mechanism [38] but scattering data would, in general, provide more direct information than $\mathrm{CID}$ spectra alone.

\section{Multiple Collisions}

Most analytical applications of MS/MS employ CA conditions that make multiple collisions likely. In beam-type instruments, for example, a trade-off is made between fragmentation efficiency and collection efficiency to maximize MS/MS efficiency [27]. The reader is referred to several studies that have focused on multiple collisions in MS/MS [28, 99-108]. The dynamics for a discrete collision have been emphasized here, and they also apply under multiple collision conditions. However, as mentioned in the section on analytical considerations, the use of multiple collisions often extends the time period over which energy is added to the ion into the time frame where chemistry (e.g., rearrangements) can occur. In some cases, this can be avoided by use of high collision energies, 
but in triple quadrupole instruments and, in particular, in ion-trapping instruments, relatively long intervals (on the order of microseconds or longer) transpire between collisions. Rearrangement of the precursor ion between collisions can occur under these circumstances [109], and that can lead to erroneous conclusions regarding the structure of the precursor ion.

The problem of rearrangement occurring prior to fragmentation in ion structural studies has long been recognized [108-111]. It is also widely recognized that this situation can be exacerbated by the use of multiple low energy activation events separated in time by up to microseconds, as in many multiple collision CA experiments. Why, then are multiple collision conditions used so extensively? For one reason, precursor ion rearrangements during ion activation do not adversely affect many analytical applications. Furthermore, most of the even-electron precursor ions typically formed from the newer ionization methods used in analytical applications probably do not rearrange as readily as odd-electron precursor ions. The main reason, however, is a practical one: product ion yields and energy deposition into the precursor ion are greater under multiple collision conditions than under single collision conditions. For precursor ions of all sizes, it has been observed repeatedly that product ion yields, up to a point, and overall energy deposition are enhanced under multiple collision conditions. These benefits usually outweigh the disadvantages associated with slow activation.

The issue of multiple versus single collision conditions is particularly relevant in the challenging area of MS/MS of high mass ions $\left(m_{p}>1000 \mathrm{u}\right)$. A widely perceived weakness of $C A$ as an activation method for high mass ions, at least in the fast ion-resting target situation, derives from eq 7 . The fraction $N /\left(N+m_{p}\right)$ becomes increasingly small as $m_{p}$ increases, thereby reducing $\mathrm{KE}_{\mathrm{rel}}$ (It should also be recognized that the relative velocity of the collision partners, and hence $t_{c}$, also decreases, thereby influencing the dynamics.) Part of the motivation behind the study of alternative activation methods is to increase $K E_{\text {rel }}$ either by using light projectiles to bombard the ion (e.g, electrons [112-116] or photons $[63,117-120])$ or by increasing the mass of the target (e.g., a solid surface [121-125]). An approach to increase $K_{\text {rel }}$ in $C A$ by bombarding the ion with fast atoms - that is, a slow ion-fast neutral approach - has also been proposed [126]. Each of these approaches has its "figures of merit" and may prove to be valuable for high mass ions. However, it is still too early to give up on CA using relatively slow targets. In doing so, the advantage of ready variability of target thickness is lost. Let us therefore consider what direction might seem to be fruitful in making $C A$ with a slow target work for high mass ions.

One approach to increasing $\mathrm{KE}_{\text {rel }}$ in the fast ion-slow target scenario is to increase $\mathrm{KE}_{\text {lab }}$. This is, of course, a brute strength approach and might require expensive and hazardous ion acceleration equipment.
A further drawback to this approach is the decreasing efficiency of $\mathrm{CA}$ with $\mathrm{KE}_{\text {rel. }}$. It has been universally observed that efficiency, $Q / \mathrm{KE}_{\mathrm{rel}}$, decreases monotonically with $K E_{\text {rel }}$. Therefore, diminishing returns accrue from increasing collision energy. The use of megaelectron-volt laboratory collision energies under single collision conditions, an alternative on one extreme, therefore, does not appear to be an attractive approach for either practical or fundamental reasons. An approach on the other extreme would be to use many high efficiency collisions of low $\mathrm{KE}_{\mathrm{rel}}$. The use of multiple collisions at electron-volt $\mathrm{KE}_{\text {lab }}$ is a step in this direction. Collisional activation in the quadrupole ion trap is perhaps the most extreme example of this type of CA in MS/MS instruments currently in use. Taking the concept further, a high temperature oven might be imagined-that is, a slow ion-slow target situation. Although not a part of an MS/MS experiment, Busman et al. have shown heating in an electrospray ionization source to be quite effective at dissociating high mass multiply charged proteins [127]. Any molecule can be dissociated by gas-phase "heating," which is what CA is. Collisional activation is most efficient at low $\mathrm{KE}_{\mathrm{rel}}$, but many collisions are needed. Current MS/MS instruments do not allow for this oven scenario between stages of MS/MS, although the quadrupole ion trap probably comes closest. Research in this direction, therefore, might hold promise for CA of high mass ions. Of course, ample opportunity for chemistry (including possible rearrangements) would be available in this approach. Perhaps the major challenge would be in extracting useful structural information from the experiment rather than in inducing fragmentation.

\section{Collisional Activation: Prospects}

Collisional activation was the first activation method used for MS /MS, and it is still by far the most widely used method. Significant effort has gone into the study of other activation methods, and many of them present advantages over CA in some respects. Some of these methods have clear benefits over CA in specific applications. However, it seems unlikely that any alternate activation method will soon supplant $C A$ in analytical MS/MS. Overall, its figures of merit are superior to those of other approaches in most analytical scenarios. Perhaps the major failing of $\mathrm{CA}_{x}$ as it is currently employed, comes in its ineffectiveness in dissociating high mass ions and ions with particularly high barriers for dissociation. There may be other approaches, however, ones that may require new types of instrumentation, that will make CA more effective for these ions. In any case, $C A$ will remain with us for the foreseeable future. Our understanding of $C A$ has improved dramatically since the 1968 papers that initiated interest in it among organic mass spectrometrists. However, much has yet to be learned about target effects, and the 
relative importance of the various mechanisms for many of the kinds of precursor ions formed by "soft" ionization methods. For this reason, research into the dynamics of CA of polyatomic ions remains particularly relevant to the progress of organic mass spectrometry.

\section{Acknowledgments}

The author expresses both gratitude to and admiration for the scientists, too many to name here, who have contributed to our understanding of collisional activation in mass spectrometry and, in particular, thanks Dr. Robert K. Boyd and Dr. Michael L. Gross for the opportunity to review this field. The author also acknowledges support from the United States Department of Energy Office of Basic Energy Sciences, under Contract DE-ACO5840R21400 with Martin Marietta Energy Systems, Inc.

\section{References}

1. Durup. J. In: Recent Developments in Mass Spectrometry; Ogata, K.; Hayakawa, T., Eds.; University of Tokyo Press: Tokyo, 1970; $\mathrm{p} 921$.

2. Cooks, R. G., Ed. Collision Spectroscopy; Plenum Press: New York, 1978.

3. Busch, K. L.; Glish, G. L; McLuckey, S. A. In: Mass Spectrometry / Mass Spectrometry: Techniques and Applications in Tandem Mass Spectrometry; VCH Publishers: New York, 1988, ch 3 .

4. Levsen, K; Schwarz, H. Mass Spectrom. Kev. 1983, $2,77$.

5. Todd, P. J.: McL Lafferty, F. W. In: Tandem Mass Spectrametry; McLafferty, F. W., Ed.; Wiley: New York, 1983; p 149

6. Dawson, P. H.; Douglas, D. J. In: Tandem Mass Spectrometry; McLafferty, F. W., Ed.; Wiley: New York, 1983; p 125.

7. Singh, S.; Harris, F. W.; Boyd, R. K.; Beynon, J. H. Int. J. Mass Spectrom. Ion Proc. 1985, 66, 131.

8. Hayes, R. N.; Gross, M. L. In: Methods in Enzymology, vol 19; Mass Spectrometry; McCloskey, J. A., Ed.; Academic Press: Orlando, 1990, ch 10

9. Kim, M. S. Org. Mass Spectrom. 1991, 26, 565.

10. Thomson, J. J. Rays of Positive Electricity and Their Application to Chemical Analysis; Longmans, Green: London, 1913.

11. Aston, F. W. Proc. Cambridge Philos. Sac. 1919, 19, 317.

12. Haddon, W. F.; McLafferty, F. W. J. Am. Chem. Soc. 1968, 90, 4745 .

13. Jennings, K. R. Int. J. Mass Spectrom. Ion Phys. 1968, 1, 227.

14. Kolotyrkin, V. M.; Tikhomirov, M. V.; Tunitskii, N. I. Doki. Akad. Nauk SSSR 1953, 92, 1193.

15. Melton, C. E.; Rosenstock, H. M. J. Chem. Phys. 1957, 26, 568.

16. Kupriyanov, S. E.; Perov, A. A. Russ. J. Phys. Chem. 1965, 39, 871.

17. McLafferty, F. W.; Schuddemage, H. D. R. J. Am. Chem. Soc. 1969, 91, 1866.

18. McLafferty, F. W.; Kornfeld, R.; Haddon, W. F.; Levsen, K.; Sakai, I.; Bente III, P. F.; Tsai, S. C.; Schuddemage, H. D. R. J. Am. Chem. Soc. 1973, 95, 3886.

19. McLafferty, F. W.; Bente III, P. F.; Kornfeld, R.; Tsai, S. C.; Howe, I. J. Am. Chem. Soc, 1973, 95, 2120.

20. Beynon, J. H.; Caprioli, R. M.; Ast, T. Int. J. Mass Spectrom. Ion Phys. 1972, 7, 88.

21. Cooks, R. G.; Hendricks, L.; Beynon, J. H. Org. Mass Spectrom. 1975, 10, 625 .

22. Kim, K. C.; Uckotter, M.; Beynon, J. H.; Cooks, R. G. Int. J. Mass Spectrom. Ion Phys. 1974, 15, 23.
23. Cooks, R. G.; Beynon, J. H.; Caprioli, R. M.; Lester, G. R. Metastable Ions; Elsevier: Amsterdam, 1973.

24. Kondrat, R. W.; Cooks, R. G. Anal. Chem. 1978, 50, 81 A.

25. Busch, K. L.; Cooks, R. G. In: Tandem Mass Spectrometry; McLafferty, F. W.; Ed.; Wiley: New York, 1983, p 11.

26. Yost, R. A.; Enke, C. G. J. Am. Chem. Soc. 1978, 100, 2274.

27. Yost, R. A.; Enke, C. G. Anal. Chem. 1979, 51, 1251 A.

28. Douglas, D. J. J. Phys. Chem. 1982, 86, 185.

29. Dawson, P. H. Int. I. Mass Spectrom. Ion Phys. 1982, 43, 195.

30. Dawson, P. H. Int. J. Mass Spectrom. Ion Phys. 1983, 50, 287.

31. Cody, R. B.; Freiser, B. S. Int. I. Mass Spectrom. Ion Phys. 1982, 41, 199.

32. Louris, J. N.; Cooks, R. G.; Syka, J. E. P.; Kelley, P. E.; Stafford, Jr., G. C.; Todd, J. F. J. Anal. Chem. 1987, 59, 1677.

33. Kim, M. S.; McLafferty, F. W. I. Am. Chem. Soc. 1978, 100, 3279.

34. Lee, S. H.; Kin, M. S.; Beynon, J. H. Int. J. Mass Spectrom. Ion Phys. 1987, 75, 83.

35. Vekey, K.; Brenton, A. G.; Beynon, J. H. J. Phys. Chem. 1986, 90,3569 .

36. Kenttämaa, H. I.; Cooks, R. G. Int. J. Mass Spectrom. Ion Proc. 1985, 64, 79.

37. Wysocki, V. H.; Kenttämaa, H. I.; Cooks, R. G. Int. J. Mass Spectrom. Ion Proc. 1987, 75, 181.

38. Horning, S. R.; Vincenti, M.; Cooks, R. G. I. Am. Chem. Soc. $1990,112,119$.

39. Dawson, P. H.; Sun, W. F. Int. I. Mass Spectrom. Ion Proc. 1983-1984, 55, 155.

40. Martinez, R. J. Rapia Commun. Mass Spectrom. 1989, 2, 127.

41. McLuckey, S. A.; Cooks, R. G. In: Tandem Mass Spectrometry; McLafferty, F. W., Ed.; Wiley: New York, 1983, p 303.

42. McLuckey, S. A.; Glish, G. L.; Cooks, R. G. Int. I. Mass Spectrom. Ion Phys. 1981, 39, 219.

43. Fetterolf, D. D; Yost, R. A. Int. I. Mass Spectrom. Ion Phys. 1982, 44, 37.

44. Nacson, S.; Harrison, A. G. Int. J. Mass Spectrom. Ion Proc. $1985,63,325$.

45. Laramee, J. A.; Carmody, J. J.; Cooks, R. G. Int. I. Mass Spectrom. Ion Phys. 1979, 31, 333.

46. Hemberger, P. H.; Laramee, J. A.; Hubik, A. R.; Cooks, R. G. J. Phys. Chem. 1981, 85, 2335.

47. McLuckey, S. A.; Verma, S.; Cooks, R. G.; Farncombe, M. J.; Mason, R. S; Jennings, K. Int. I. Mass Spectrom. Ion Proc. $1983,48,423$.

48. Todd, P. J.; Warmack, R. J.; McBay, E. H. Int. J. Mass Spectrom. Ion Proc. 1983, 50, 299.

49. Boyd, R. K.; Kingston, E. E.; Brenton, A. G.; Beynon, J. H. Proc. R. Sac. London (A) 1984, 392, 59

50. Waddell, R. K.; Boyd, R. K.; Brenton, A. G.; Beynon, J. H. Int. J. Mass Spectrom. Ion Proc. 1986, 68, 71.

51. Singh, S.; Harris, F. W.; Boyd, R. K.; Beynon, J. H. Int. J. Mass Spectrom. Ion Proc. 1985, 66, 151.

52. Van Der Zande, W. J.; De Bruijn, D. P.; Los, J.; Kistemaker, P. G.; McLuckey, S. A. Int. J. Mass Spectrom. Ion Prac. 1985, 67, 161.

53. Reid, C. J. Org. Mass Spectrom. 1989, 24, 405.

54. Reid, C. J. J. Phys. (B) 1990, 23, 2783.

55. Reid, C. J. Org. Mass Spectrom. 1991, $26,402$.

56. Reid, C. J.; Ballantine, J. A. Int. I. Mass Spectrom. Ion Proc. 1991, 105, 161.

57. Reid, C. J. Int. J. Mass Spectrom. Ion Proc. 1991, 105, 191.

58. Cooks, R. G.; Mabud, A.; Horning, S. R.; Jiang, X. Y.; Paradisi, C.; Traldi, P. J. Am. Chem. Soc. 1989, 111, 859. 
59. Burinsky, D. J.; Glish, G. L.; Cooks, R. G.; Zwinselman, J. J.; Nibbering, N. M. M. J. Am. Chem. Soc. 1981, 103, 465.

60. Reid, C. J.; Harris, F. M. Int. J. Mass Spectrom. Ion Proc. 1988, 85, 151.

61. Vincenti, M.; Horning, S. R, Cooks, R. G. Org. Mass Spectrom. 1988, 23, 585.

62. Brodbelt-Lustig, J. S.; Cooks, R. G. Int. J. Mass Spectrom. Ion Proc. 1988, 86, 253.

63. Thorne, L. R; Beauchamp, J. L. In: Gas Phase Ion Chemistry; Bowers, M. T., Ed.; Academic Press: Orlando, 1984, vol 3.

64. McLuckey, S. A.; Cooks, R. G. Int. J. Mass Spectrom. Ion Proc. 1984, 56, 223.

65. Shirts, R. B. In: Gaseous Ion Chemistry and Mass Spectrometry; Futrell, J. H., Ed.; Wiley: New York, 1986, ch 2.

66. Mahan, B. H. J. Chem. Phys. 1970, 52, 5221.

67. Futrell, J. H. In: Gaseous Ion Chemistry and Mass Spectrometry; Futrell, J. H., Ed.; Wiley: New York, 1986, ch 10.

68. Gentry, W. R. In: Gas Phase Ion Chemistry; Bowers, M. T., Ed.; Academic Press: Orlando, 1979, vol 2.

69. Herman, Z.; Futrell, J. H.; Friedrich, B. Int. J. Mass Spectrom. Ion Proc. 1984, 58, 299

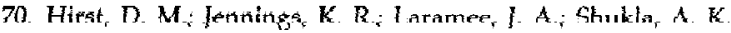

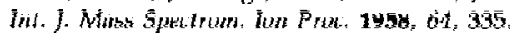

71. Shukla, $\Lambda$. K.; Anderson, S. G.; Howard, 5. L.; Sohlberg, K. W.; Futrell, J. H. Int. J. Mass Spectrom. Ion Froc. 1988, 86, 61.

72. Shukla, A. K.; Qian, K.; Howard, S. L; Anderson, S. G.; Sohlberg, K. W.; Futrell, J. H. Int. J. Mass Spectrom lon Proc. 1989, 92, 147.

73. Qian, K.; Shukla, A. K.; Howard, S. L.; Anderson, S. G.; Futrell, J. H. J. Phys. Chem. 1989, 93, 3889.

74. Shukla, A. K.; Qian, K.; Anderson, S. G; Futrell, J. H. J. Am. Soc. Mass Spectrom. 1990, 1, 6.

75. Everhart, E. Phys. Rev. 1963, 132, 2083.

76. Smith, F. T.; Marchi, R. P.; Dedrick, K. G. Phys. Rev. 1966, $150,79$.

77. Knudhausen, E.; Pauly, H. Z. Phys. 1965, 187, 305.

78. Aue, D. H; Bowers, M. T. In: Gas Phase Ion Chemistry; Bowers, M. T., Ed.; Academic Press: Orlando, 1979, vol 2.

79. Massey, H. S. W. Rep. Prog. Phys. 1949, 12, 3279

80. Hasted, J. B.; Lee, A. R. Proc. Phys. Soc. 1962, 79, 702.

81. Orlando, R.; Fenselau, C.; Cotter, R. J. J. Am. Soc. Mass Spectrom. 1991, 2, 189.

82. Meot-Ner, M. In: Gas Phase Ion Chemistry; Bowers, M. T., Ed.; Academic Press: Orlando, 1979, vol 1.

83. Mahan, B. H. Acc. Chem. Res. 1970, 3, 393.

84. Levine, R. D; Bernstein. R. B. Molecular Reaction Dynamics; Oxford University Press: New York, 1974, ch 6.

85. Forst, W. Theory of Unimolecular Reactions; Academic Press: Orlando, 1973, ch 2.

86. Alexander, A. J.; Boyd, R. K. Int. J. Mass Spectrom. Ion Proc. $1989,90,211$

87. Gilbert, R. G.; Sheil, M. M.; Derrick, P. J. Org. Mass Spectrom $1985,20,430$.

88. Neumann, G. M.; Derrick, P. J. Org. Mass Spectrom. 1984, 19 , 165.

89. Uggerud, E.; Derrick, P. J. Z. Naturforsch. 1989, 44a, 245.

90. Fernandez, S. M.; Erikson, F. J.; Bray, A. V.; Pollack, E. Phys. Rev. (A) 1975, 12, 1252.

91. Anderson, N.; Vedder, M.; Russek, A.; Pollack, E. Phys. Rev. (A) 1980, 21, 782 .

92. Russek, A. Physica, 1970, 48, 165

93. Qian, K.; Shukla, A. K.; Futrell, J. H. Rapid Commun. Mass Spectrom. 1990, 4, 222.
94. Shukla, A. K.; Futrell, J. H. Proceedings of the 39th ASMS Conference on Mass Spectrometry and Allied Topics, Nashville, TN, May 20-24, 1991.

95. Shukla, A. K. Rapid Commun. Mass Spectrom. 1990, 4, 137.

96. Laramee, J. A.; Cameron, D.; Cooks, R. G. I. Am. Chem. Soc. 1981, 103, 12.

97. Bricker, D. L.; Russell, D. H. J. Am. Chem. Soc. 1986, 108, 6174.

98. Ouwerkerk, C. E. D.; McLuckey, S. A.; Kistemaker, P. G.; Boerboom, A. J. H. Int. I. Mass Spectrom. Ion Proc. 1984, 56, 11.

99. Boyd, R. K. Int. 1. Mass Spectrom. Ion Proc. 1987, 75, 243.

100. Gross, M. L.; Tomer, K. B.; Cerny, R. L.; Giblin, D. E. In: Mass Spectrometry in the Analysis of Large Molecules; McNeal, C. J., Ed.; Wiley: New York, 1986.

101. Nystrom, J. A.; Bursey, M. M.; Hass, J. R. Int. J. Mass Spectrom. Ion Proc. 1983-1984, 55, 263.

102. Bursey, M. M.; Nystrom, J. A.; Hass, J. R. Anal. Chim. Acta 1984, 159, 265.

103. Schey, K. L.; Kenttämaa, H. I.; Wysocki, V. H.; Cooks, R. G. Int. J. Mass Spectrom. Ion Proc. 1989, 90, 71.

104. Alexander, A. J.; Thibault, P.; Boyd, R. K. I. Am. Chem. Soc. $1990,112,24 B 4$

105. Todt, F. J.; MoLafferty, F. W. Int. I. Muss Spuinum. Ion Fhys. $1981,38,3 \overline{7} 1$.

106. Kim, M. S. Int. J. Mazss Spectrom. Ion Phys. 1983, 50, 189.

107. Kim, M. S. int. J. Mase Spectront. fon Pitys. 1983, 51, 279.

108. Holmes, J. I. Org. Mass Spectrom. 1985, 20, 169.

109. Kentämaa, H. I.; Cooks, R. G. I. Am. Chem. Soc. 1985, 107, 1881.

110. McLafferty, F. W. Philos. Trans. R. Soc. Lond. (A) 1979, 293, 93.

111. Levsen, K. Fundamental Aspects of Organic Mass Spectrometry; Springer-Verlag: Weinheim, 1978.

112. Cody, R. B.; Freiser, B. S. Anal. Chem. 1987, 59, 1056.

113. Cody, R. B.; Freiser, B. S. Anal. Chem. 1979, 51, 546.

114. Shulz, P. A.; Gregory, D. C.; Meyer, F. W.; Phaneuf, R. A. J. Chem. Phys. 1986, 85, 3386.

115. Tajima, S.; Tobita, S.; Ogino, K.; Niwa, Y. Org. Mass Spectrom. 1986, 21, 236

116. Aberth, W.; Burlingame, A. L. In: Biological Mass Spectrometry; Burlingame; A. L.; McCloskey, I. A., Eds.; Elsevier: Amsterdam, 1990, p 217.

117. Russell, D. H. Mass Spectrom. Rev. 1990, 9, 405

118. Dunbar, R. C. In; Gas Phase Ion Chemistry; Bowers, M. T., Ed.; Academic Press: Orlando, 1979, vol 2, ch 14.

119. Dunbar, R. C. In: Gas Phase Ion Chemistry; Bowers, M. T., Ed.; Academic Press: Orlando, 1984, vol 3, ch 20.

120. Harris, F, M, Beynon, J. H. In; Gas Phase Ion Chemistry; Bowers, M. T., Ed.; Academic Press: Orlando, 1984, vol 3, ch 19.

121. Mabud, M. A.; De Krey, M. J.; Cooks, R. G. Int. J. Mass Spectrom. Ion Proc. 1986, 21, 193.

122. De Krey, M. J.; Mabud, M. A.; Cooks, R. G.; Syka, J. E. P. Int. J. Mass Spectrom. Ion Proc. 1985, 67, 295.

123. De Krey, M. J.; Kenttämaa, H. I.; Wysocki, V. H.; Cooks, R. G. Org. Mass Spectrom 1986, 21, 193.

124. Schey, K.; Cooks, R. G.; Grix, R.; Wollnik, H. Int. I. Mass Spectrom. Ion Proc. 1987, 77, 49

125. Cooks, R. G.; Ast, T.; Mabud, M. A. Int. J. Mass Spectrom. Ion Proc. 1990, 100, 209.

126. Turecek, F. Rapid Commun. Mass Spectrom. 1991, 5, 78.

127. Busman, M.; Rockwood, A. L.; Ud seth, H. R.; Smith, R. D. Proceedings of the 39th ASMS Conference on Mass Spectrometry and Allied Topics, Nashville, TN, May 20-24, 1991. 